\title{
Utilização dos Equipamentos de Proteção Individual pela equipe de enfermagem no cenário pandêmico
}

Use of Personal Protective Equipment by the nursing staff in the pandemic scenario

Uso de Equipo de Protección personal por parte del personal de enfermería en el escenario de una pandemia

Recebido: 06/01/2022 | Revisado: 11/01/2022 |Aceito: 15/01/2022 | Publicado: 17/01/2022

\author{
Rafaela São Miguel de Souza \\ ORCID: https://orcid.org/0000-0002-3718-2173 \\ Universidade Federal de Rondonópolis, Brasil \\ E-mail: rafaelasaomiguel@hotmail.com \\ Graciela da Silva Migueis \\ ORCID: https://orcid.org/0000-0001-7287-6026 \\ Universidade Federal de Rondonópolis, Brasil \\ E-mail: graciela.migueis@ufr.edu.br \\ Wanmar de Souza Oliveira \\ ORCID: https://orcid.org/0000-0002-7233-4777 \\ Universidade Federal de Rondonópolis, Brasil \\ E-mail:w-anmar@hotmail.com \\ Michele Salles da Silva \\ ORCID: https://orcid.org/0000-0002-4076-5628 \\ Universidade Federal de Rondonópolis, Brasil \\ E-mail: michele.salles@ufr.edu.br \\ Vanessa Alves Mendes \\ ORCID: https://orcid.org/0000-0003-2284-5082 \\ Universidade Federal de Rondonópolis, Brasil \\ E-mail: vanessa.mendes@ufr.edu.br
}

\begin{abstract}
Resumo
SARS-CoV-2 é responsável por causar a doença Covid-19, com alto grau de contágio e propagação rápida. A contaminação se dá através do contato com gotículas, aerossóis e outras secreções, esse contato pode ser por meio direto ou indireto e com manifestações de sintomas leves a graves. Os profissionais de enfermagem possuem maior risco de serem contaminados, visto que, mantêm contato contínuo e integral com os pacientes hospitalizados, contudo, o uso de Equipamento Proteção Individual (EPI) é uma das medidas eficientes na diminuição da contaminação entre esse público, caso a utilizem de maneira correta. O presente estudo teve por objetivo descrever o conhecimento dos profissionais de enfermagem sobre o uso de EPI necessários ao cuidado do paciente acometido pela Covid-19. Tratase de um estudo quantitativo, descritivo, do tipo transversal, realizado em um hospital no interior de Mato Grosso. Foram entrevistados 50 profissionais atuantes na linha de frente a Covid-19, sendo 7 enfermeiros e 43 técnicos em Enfermagem. Os achados mostram de modo geral que os profissionais utilizam os EPI em procedimentos desde a triagem até àqueles geradores de aerossóis. Os EPI com maior citação de uso foram: luvas de procedimento, máscara N95 e avental descartável. Em síntese, a utilização do EPI precisa ser aderida entre os profissionais durante alguns procedimentos.
\end{abstract}

Palavras-chave: Equipamento de Proteção Individual; Covid-19; Enfermagem.

\begin{abstract}
SARS-CoV-2 is responsible for causing the Covid-19 disease, with a high degree of contagiousness and rapid spread. Contamination occurs through contact with droplets, aerosols and other secretions, and this contact can be direct or indirect, with manifestations of mild to severe symptoms. However, the use of Personal Protective Equipment (PPE) is one of the most efficient measures to reduce contamination among this public, if used correctly. The present study aimed to describe the knowledge of nursing professionals about the use of PPE needed to care for patients affected by Covid-19. This is a quantitative, descriptive, cross-sectional study, carried out in a hospital in the interior of Mato Grosso. Foram entrevistados 50 profissionais atuantes na linha de frente a Covid-19, sendo 7 enfermeiros e 43 técnicos em Enfermagem. The findings show that in general the professionals use PPE in procedures from triage to those generating aerosols. The PPE most often mentioned were: procedure gloves, N95 mask, and disposable apron. In summary, the use of PPE needs to be adhered to among professionals during some procedures.
\end{abstract}

Keywords: Personal Protective Equipment; Covid-19; Nursing. 


\begin{abstract}
Resumen
El SARS-CoV-2 es responsable de causar la enfermedad Covid-19, con un alto grado de contagio y una rápida propagación. La contaminación se produce por el contacto con gotitas, aerosoles y otras secreciones, y este contacto puede ser directo o indirecto, con manifestaciones de síntomas leves a graves. Sin embargo, el uso de equipos de protección individual (EPI) es una de las medidas más eficaces para reducir la contaminación entre este público, si se utiliza correctamente. El presente estudio pretendía describir los conocimientos de los profesionales de enfermería sobre el uso de los EPI necesarios para atender a los pacientes afectados por Covid-19. Se trata de un estudio cuantitativo, descriptivo, transversal, realizado en un hospital del interior de Mato Grosso. Se entrevistó a 50 profesionales que actúan en primera línea ante Covid-19, siendo 7 enfermeras y 43 técnicos de enfermería. Los resultados muestran que, en general, los profesionales utilizan los EPI en procedimientos que van desde el triaje hasta los generadores de aerosoles. Los EPI más mencionados fueron: guantes de procedimiento, mascarilla N95 y delantal desechable. En resumen, es necesario que los profesionales respeten el uso de los EPI durante algunos procedimientos.
\end{abstract}

Palabras clave: Equipos de protección individual; Covid-19; Enfermería.

\title{
1. Introdução
}

O SARS-CoV-2, também conhecido coronavírus, responsável por causar a doença Covid-19, se originou em Wuhan, província de Hubei na China em dezembro de 2019. O vírus possui grau de contágio elevado e propagação rápida. Em 11 de março de 2020, a Covid-19 foi caracterizada pela Organização Mundial de Saúde (OMS) como uma pandemia. A contaminação se dá através do contato com gotículas, aerossóis e outras secreções, esse contato pode ser por meio direto ou indireto. $\mathrm{O}$ contato indireto ocorre quando a pessoa toca uma superfície contaminada e acaba se expondo ao vírus, já no contato direto, a pessoa que esteja a menos de 1 metro de outra pessoa contaminada corre o risco de entrar em contato com gotículas e secreções expelidas no momento da fala, do ato de rir, tossir ou espirrar (WHO, 2020a).

Alguns sintomas clínicos da doença são: tosse, dor de garganta, dor de cabeça, febre $\geq 37,8^{\circ} \mathrm{C}$, fadiga, diarreia, anosmia (perda do olfato) ou hiposmia (diminuição do olfato), ageusia (perda do sentido do paladar), isso em casos leves. Já em quadros mais graves, o paciente pode apresentar todos os sintomas anteriores, juntamente com pneumonia, que é capaz de levar a um quadro de síndrome respiratória aguda podendo levar a óbito (Antunes et al., 2021).

Nesse contexto, a vigilância epidemiológica auxilia na prevenção, na identificação de casos suspeitos e/ ou confirmados e na implementação de medidas preventivas que diminuem o risco de exposição e a consequente contaminação pelo novo vírus (Carvalho et al., 2021). Em vista disso, o isolamento social, o uso da máscara e a higienização correta das mãos foram implementadas como medidas eficientes na diminuição das chances de contaminação entre a população.

Contudo, há pessoas de várias áreas de atuação, que atuam na linha de frente, não conseguindo adotar o isolamento rigoroso, uma vez que, não podem parar de desempenhar seu papel na sociedade, dentre esses destaca-se a área da saúde. Segundo Antunes et al. (2021), os profissionais de saúde, mais precisamente os enfermeiros, técnicos e auxiliares de enfermagem, possuem maior risco de serem contaminados, visto que mantêm contato contínuo e integral com os pacientes hospitalizados.

Particularmente, além das medidas necessárias para não se contaminar com o Covid-19, como, lavagem das mãos e o uso de álcool 70\%, os profissionais da saúde têm como aliado o Equipamento de Proteção Individual (EPI). EPI conforme Norma Regulamentadora (NR6), é o aparato empregue para os colaboradores de uma instituição, com intuito de preservar e proporcionar segurança a eles. A NR6 menciona que as organizações devem propiciar tais equipamentos gratuitamente, com qualidade e sem falhas, levando em conta grau de periculosidade de cada área e momento de atuação (Brasil, 2020a).

Considera-se os EPIs a principal medida preventiva implementada nas diferentes áreas de atuação do cuidado em saúde (Carvalho et al., 2021). Estes devem ser utilizados em todos os momentos da assistência, a fim de garantir a segurança, pois na medida em que agem como barreira de proteção contra patógenos, diminuem as chances de contágio pelo vírus (Tristão $\&$ Tavares, 2020). 
Dessa maneira, na assistência ao paciente, em cada procedimento há um equipamento de proteção adequado a ser utilizado, e se faz de extrema importância que a equipe de enfermagem conheça cada um deles e saiba o momento adequado para o seu uso. Contudo, atualmente o contexto de pandemia, impôs uma nova rotina em seu cotidiano de trabalho permeado por uma afecção de alta virulência e contágio, o que requer adequada atenção no manuseio/uso do EPI para evitar contaminação. Segundo Oliveira et al. (2021), a situação pandêmica acarreta incertezas, estresse, muitas vezes com sobrecarga de trabalho, esgotamento mental, cansaço físico e condições de trabalho inadequadas, situações que podem interferir na assistência de qualidade. Acrescentamos, que esta situação pode trazer consequências ao profissional caso o uso do EPI não seja adequado.

A enfermagem tem se apresentado como o grupo profissional de maior contaminação pelo SARS-CoV-2. Os profissionais de saúde envolvidos direta e indiretamente no enfrentamento da pandemia estão expostos cotidianamente ao risco de adoecer pelo coronavírus, sendo que a heterogeneidade que caracteriza este contingente da força de trabalho determina formas diferentes de exposição, tanto ao risco de contaminação quanto aos fatores associados às condições de trabalho, mesmo quando protegidos adequadamente (Teixeira et al., 2020).

Nesse contexto, diante de uma nova realidade imposta pelo coronavírus, se faz necessário avaliar os saberes dos trabalhadores acerca do EPI. Dessa forma, questiona-se, os profissionais de saúde frente à pandemia estão manuseando corretamente os EPI?

Nessa perspectiva, o presente estudo se mostra relevante visto que agrega conhecimento acerca do uso dos EPI pelos profissionais de enfermagem em suas assistências prestadas. Os achados nesta pesquisa irão permitir problematizar a realidade local, bem como avaliar os saberes dos trabalhadores acima do tema contemplado. Além disso, visa contribuir com a comunidade científica e sociedade na socialização de conhecimento inovador direcionado para o cenário pandêmico atual. Assim, o estudo teve por objetivo caracterizar a utilização dos equipamentos de proteção individual por profissionais de enfermagem durante o cuidado ao paciente acometido pela Covid-19.

\section{Metodologia}

Trata-se de um estudo quantitativo, descritivo, do tipo transversal, realizado em um hospital no interior de Mato Grosso. A pesquisa quantitativa é uma modalidade de pesquisa que atua sobre um problema humano ou social, composta por variáveis quantificadas em números, as quais são analisadas de modo estatístico, a fim de determinar se as generalizações previstas na teoria se sustentam ou não (Knechtel, 2014).

Foi realizada a aplicação de questionário estruturado com a equipe de enfermagem atuantes nos setores de assistência destinada ao atendimento de pacientes por Covid-19, sendo: Unidade de Terapia Intensiva (UTI) Geral, UTI Covid e Enfermaria Covid. Para atender aos objetivos propostos na pesquisa, foram utilizados como critérios de inclusão: ser profissional de enfermagem com vínculo empregatício com a Instituição hospitalar; estar lotado em uma das unidades de assistência ao paciente com Covid-19; prestar assistência direta aos pacientes com Covid-19 e ser maior de idade. Foram considerados inelegíveis para a participação da pesquisa, os profissionais que estavam no período de férias, licença saúde ou ausente do setor após três tentativas de contato no decorrer da coleta de dados, portanto, excluídos da coleta.

A coleta de dados foi realizada presencialmente no turno de trabalho diurno e noturno dos colaboradores da instituição, sendo sempre agendada com antecedência para não influenciar na rotina de trabalho. A entrevista foi realizada individualmente e seguindo as recomendações sanitárias vigentes para a Covid-19, além disso, os participantes assinaram o Termo de Consentimento Livre e Esclarecido. 
O instrumento, tipo questionário, foi composto por duas partes: uma para caracterização dos participantes e a outra sobre o conhecimento quanto ao uso do EPI no atendimento ao paciente com Covid-19. Os dados foram coletados de agosto de 2020 a janeiro de 2021.

Após a coleta, os dados foram organizados em uma planilha com o auxílio do software Microsoft Office Excel®, realizados cálculos de frequência simples e absoluta. Posteriormente analisados de maneira descritiva e apresentados na forma de gráficos e tabela.

O estudo em questão é um recorte de uma pesquisa matricial que fora submetida à apreciação do Comitê de Ética em Pesquisa e recebeu parecer favorável sob n. CAAE 33496120.1.0000.8088.

\section{Resultados}

A pesquisa apontou a participação de 50 profissionais de enfermagem, 7 enfermeiros, correspondendo a $14 \%$ e 43 técnicos de enfermagem, $86 \%$. A maioria dos participantes, correspondendo a $88 \%$, são do sexo feminino e somente $12 \%$ do sexo masculino, com faixa etária predominante de 34 a 39 anos, sendo o intervalo de idade de 22 a 55 anos.

A pesquisa ocorreu em três setores de atendimentos à pacientes acometidos por Covid-19, com a seguinte participação dos profissionais de enfermagem, 22 (44\%) atuavam na UTI Covid, 16 (32\%) na Enfermaria Covid e 12 (24\%) na UTI Geral, conforme se observa no Gráfico 1 a seguir.

Gráfico 1: Caracterização dos profissionais de enfermagem atuantes no atendimento a Covid-19 quanto à gênero, idade, categoria profissional e área de atuação no município de Rondonópolis, Mato Grosso, Brasil, 2021.

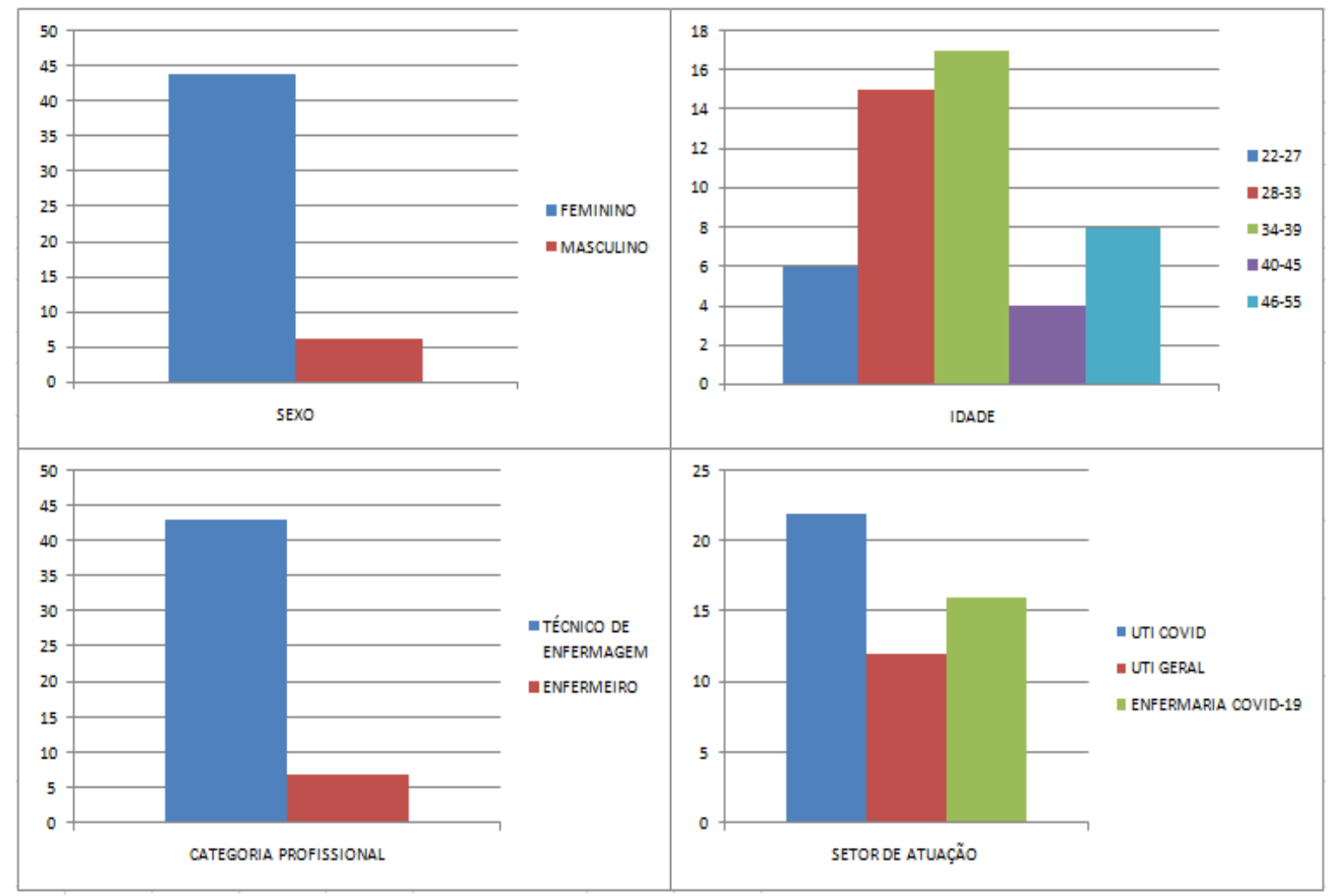

Fonte: Dados da pesquisa (2021).

O Gráfico 1 retrata a caracterização dos participantes em relação ao sexo, idade, categoria profissional e setor de atuação desses profissionais. Houve maior participação dos profissionais do setor de UTI Covid, seguido da Enfermaria Covid. 
O hospital onde foi realizada a pesquisa se reorganizou com leitos exclusivos em UTI e enfermaria para o atendimento de casos de Covid-19.

No tocante a escolaridade dos profissionais, 29 (58\%) referiram possuir o ensino médio completo, alguns possuem o ensino superior incompleto e outros são pós-graduados, este achado pode ser observado no gráfico 2 abaixo:

Gráfico 2: Escolaridade dos profissionais de Enfermagem atuantes no atendimento a Covid-19. Rondonópolis, Mato Grosso, Brasil, 2021.

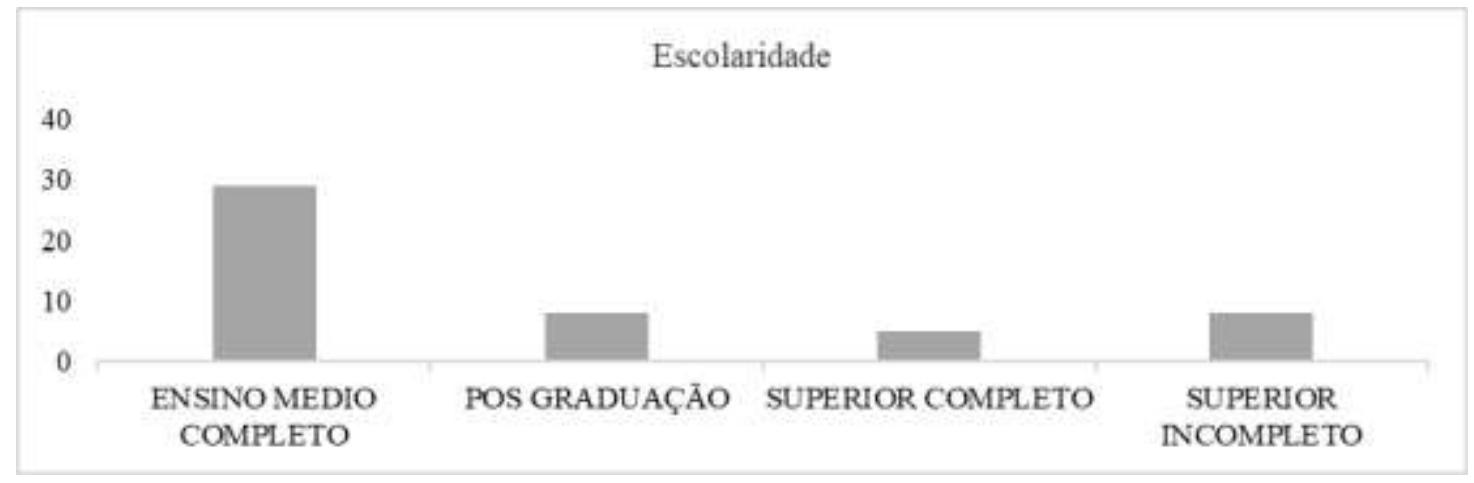

Fonte: Dados da pesquisa (2021).

O Gráfico 2 acima, é possível entender a descrição da escolaridade de acordo com sua instrução, onde mais da metade referiu possuir o ensino médio, 8 (16\%) referiu possuir ensino superior incompleto e pós-graduação respectivamente.

Em relação ao tempo de formação profissional, 18 (36\%) são formados há mais de 5 anos, 9 (18\%) são formados há menos de 6 meses e, na mesma proporção, os formados com 2 a 3 anos e 6 participantes (12\%) são formados entre 1 e 2 anos. Ademais, quanto à experiência em serviço, 17 (34\%) exercem a profissão há mais de 5 anos, em que 12 (24\%) estão há 6 meses empregados, como demonstrado no Gráfico 3 a seguir.

Gráfico 3: Tempo de formação profissional e tempo de experiência profissional dos profissionais de enfermagem atuantes no atendimento a Covid-19. Rondonópolis, Mato Grosso, Brasil, 2021.

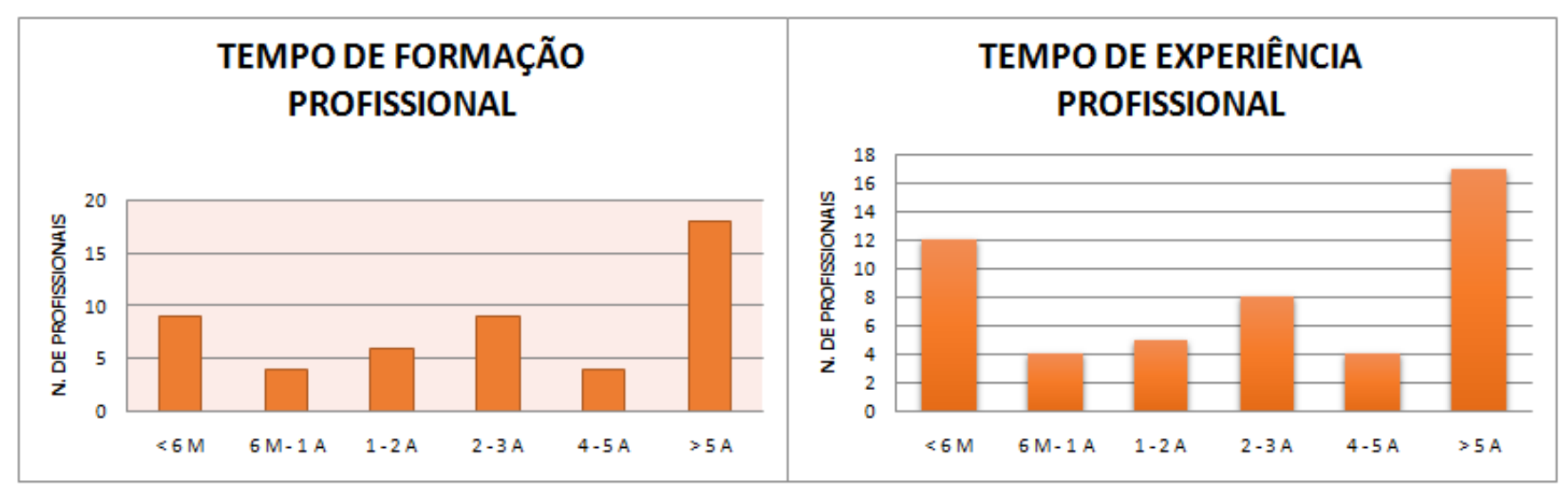

Fonte: Dados da pesquisa (2021).

Foi possível evidenciar os aspectos relacionados ao uso de EPI por procedimentos na assistência contra à Covid-19, conforme demonstrado na Tabela 1 a seguir. 
Quadro 1: EPI utilizado em cada procedimento na assistência ao paciente com Covid-19. Rondonópolis, Mato Grosso, Brasil, 2021.

\begin{tabular}{|c|c|c|c|c|c|c|c|c|c|c|c|c|c|c|c|c|c|c|c|c|}
\hline \multirow{3}{*}{$\begin{array}{l}\text { EPI utilizado } \\
\text { em cada } \\
\text { procedimento }\end{array}$} & \multicolumn{2}{|c|}{$\begin{array}{c}\text { Luvas de } \\
\text { procedimento }\end{array}$} & \multicolumn{2}{|c|}{$\begin{array}{l}\text { Máscara } \\
\text { cirúrgica }\end{array}$} & \multicolumn{2}{|c|}{$\begin{array}{c}\text { Máscara } \\
\text { N } 95\end{array}$} & \multicolumn{2}{|c|}{$\begin{array}{l}\text { Máscara } \\
\text { PFF }\end{array}$} & \multicolumn{2}{|c|}{$\begin{array}{c}\text { Máscara } \\
\text { Face Shield }\end{array}$} & \multicolumn{2}{|c|}{$\begin{array}{c}\text { Avental de } \\
\text { tecido }\end{array}$} & \multicolumn{2}{|c|}{$\begin{array}{c}\text { Avental } \\
\text { descartável }\end{array}$} & \multicolumn{2}{|c|}{$\begin{array}{c}\text { Avental } \\
\text { impermeável }\end{array}$} & \multicolumn{2}{|c|}{$\begin{array}{l}\text { Óculos de } \\
\text { proteção }\end{array}$} & \multicolumn{2}{|c|}{ Distanciamento } \\
\hline & SIM & NÃO & SIM & NÃO & SIM & NÃO & SIM & NÃO & SIM & NÃO & SIM & NÃO & SIM & NÃO & SIM & NÃO & SIM & NÃO & SIM & NÃO \\
\hline & $\begin{array}{l}\mathbf{N}^{\circ} \\
\%\end{array}$ & $\begin{array}{l}\mathbf{N}^{\circ} \\
\%\end{array}$ & $\begin{array}{l}\mathbf{N}^{\circ} \\
\%\end{array}$ & $\begin{array}{l}\mathbf{N}^{\circ} \\
\%\end{array}$ & $\begin{array}{l}\mathbf{N}^{\circ} \\
\%\end{array}$ & $\begin{array}{l}\mathbf{N}^{\circ} \\
\%\end{array}$ & $\begin{array}{l}\mathbf{N}^{\circ} \\
\%\end{array}$ & $\begin{array}{l}\mathbf{N}^{\circ} \\
\%\end{array}$ & $\begin{array}{l}\mathbf{N}^{\circ} \\
\%\end{array}$ & $\begin{array}{l}\mathbf{N}^{\circ} \\
\%\end{array}$ & $\begin{array}{l}\mathbf{N}^{\circ} \\
\%\end{array}$ & $\begin{array}{l}\mathbf{N}^{\circ} \\
\%\end{array}$ & $\begin{array}{l}\mathbf{N}^{\circ} \\
\%\end{array}$ & $\begin{array}{l}\mathbf{N}^{\circ} \\
\%\end{array}$ & $\begin{array}{l}\mathbf{N}^{\circ} \\
\%\end{array}$ & $\begin{array}{l}\mathbf{N}^{\circ} \\
\%\end{array}$ & $\begin{array}{l}\mathbf{N}^{\circ} \\
\%\end{array}$ & $\begin{array}{l}\mathbf{N}^{\circ} \\
\%\end{array}$ & $\begin{array}{l}\mathbf{N}^{\circ} \\
\%\end{array}$ & $\begin{array}{l}\mathbf{N}^{\circ} \\
\%\end{array}$ \\
\hline & 45 & 5 & 20 & 30 & 32 & 18 & 15 & 35 & 25 & 25 & 23 & 27 & 36 & 14 & 19 & 31 & 40 & 10 & 9 & 41 \\
\hline Admissāo & 90 & 10 & 40 & 60 & 64 & 36 & 30 & 70 & 50 & 50 & 46 & 54 & 72 & 28 & 38 & 62 & 80 & 20 & 18 & 82 \\
\hline Banho & $\begin{array}{l}43 \\
86\end{array}$ & $\begin{array}{c}7 \\
14\end{array}$ & $\begin{array}{l}16 \\
32\end{array}$ & $\begin{array}{l}34 \\
68\end{array}$ & $\begin{array}{l}34 \\
68\end{array}$ & $\begin{array}{l}16 \\
32\end{array}$ & $\begin{array}{l}11 \\
22\end{array}$ & $\begin{array}{l}39 \\
78\end{array}$ & $\begin{array}{l}22 \\
44\end{array}$ & $\begin{array}{l}28 \\
56\end{array}$ & $\begin{array}{l}19 \\
38\end{array}$ & $\begin{array}{l}31 \\
62\end{array}$ & $\begin{array}{l}29 \\
58\end{array}$ & $\begin{array}{l}21 \\
42\end{array}$ & $\begin{array}{l}21 \\
42\end{array}$ & $\begin{array}{l}29 \\
58\end{array}$ & $\begin{array}{l}39 \\
78\end{array}$ & $\begin{array}{l}11 \\
22\end{array}$ & - & - \\
\hline Punção venosa & $\begin{array}{l}46 \\
92\end{array}$ & $\begin{array}{l}4 \\
8\end{array}$ & $\begin{array}{l}11 \\
22\end{array}$ & $\begin{array}{l}39 \\
78\end{array}$ & $\begin{array}{l}32 \\
64\end{array}$ & $\begin{array}{l}18 \\
36\end{array}$ & $\begin{array}{l}12 \\
24\end{array}$ & $\begin{array}{l}38 \\
76\end{array}$ & $\begin{array}{l}16 \\
32\end{array}$ & $\begin{array}{l}34 \\
68\end{array}$ & $\begin{array}{l}14 \\
28\end{array}$ & $\begin{array}{l}36 \\
72\end{array}$ & $\begin{array}{l}29 \\
58\end{array}$ & $\begin{array}{l}21 \\
42\end{array}$ & 12 & $\begin{array}{l}38 \\
76\end{array}$ & $\begin{array}{l}35 \\
70\end{array}$ & $\begin{array}{l}15 \\
30\end{array}$ & - & - \\
\hline $\begin{array}{c}\text { Auxílio na } \\
\text { Intubação Oro } \\
\text { Traqueal }\end{array}$ & $\begin{array}{l}30 \\
60\end{array}$ & $\begin{array}{l}20 \\
40\end{array}$ & $\begin{array}{c}7 \\
14\end{array}$ & $\begin{array}{l}43 \\
86\end{array}$ & $\begin{array}{l}18 \\
36\end{array}$ & $\begin{array}{l}32 \\
64\end{array}$ & $\begin{array}{c}9 \\
18\end{array}$ & $\begin{array}{l}41 \\
82\end{array}$ & $\begin{array}{l}15 \\
30\end{array}$ & $\begin{array}{l}35 \\
70\end{array}$ & $\begin{array}{l}12 \\
24\end{array}$ & $\begin{array}{l}38 \\
76\end{array}$ & $\begin{array}{l}15 \\
30\end{array}$ & $\begin{array}{l}35 \\
70\end{array}$ & $\begin{array}{l}13 \\
26\end{array}$ & $\begin{array}{l}37 \\
74\end{array}$ & $\begin{array}{l}24 \\
48\end{array}$ & $\begin{array}{l}26 \\
52\end{array}$ & - & - \\
\hline Aspiração & $\begin{array}{l}27 \\
54\end{array}$ & $\begin{array}{l}23 \\
46\end{array}$ & $\begin{array}{l}4 \\
8\end{array}$ & $\begin{array}{l}46 \\
92\end{array}$ & $\begin{array}{l}23 \\
46\end{array}$ & $\begin{array}{l}27 \\
54\end{array}$ & $\begin{array}{l}4 \\
8\end{array}$ & $\begin{array}{l}46 \\
92\end{array}$ & $\begin{array}{l}13 \\
26\end{array}$ & $\begin{array}{l}37 \\
74\end{array}$ & $\begin{array}{l}10 \\
20\end{array}$ & $\begin{array}{l}40 \\
80\end{array}$ & $\begin{array}{l}16 \\
32\end{array}$ & $\begin{array}{l}34 \\
68\end{array}$ & $\begin{array}{c}9 \\
18\end{array}$ & $\begin{array}{l}41 \\
82\end{array}$ & $\begin{array}{l}23 \\
46\end{array}$ & $\begin{array}{l}27 \\
54\end{array}$ & - & - \\
\hline $\begin{array}{c}\text { Administração } \\
\text { de } \\
\text { medicamentos }\end{array}$ & $\begin{array}{l}42 \\
84\end{array}$ & $\begin{array}{c}8 \\
16\end{array}$ & $\begin{array}{l}10 \\
20\end{array}$ & $\begin{array}{l}40 \\
80\end{array}$ & $\begin{array}{l}28 \\
56\end{array}$ & $\begin{array}{c}22 \\
44\end{array}$ & $\begin{array}{l}12 \\
24\end{array}$ & $\begin{array}{l}38 \\
76\end{array}$ & $\begin{array}{l}16 \\
32\end{array}$ & $\begin{array}{l}34 \\
68\end{array}$ & $\begin{array}{l}18 \\
36\end{array}$ & $\begin{array}{l}31 \\
62\end{array}$ & $\begin{array}{l}21 \\
42\end{array}$ & $\begin{array}{l}29 \\
58\end{array}$ & $\begin{array}{l}13 \\
26\end{array}$ & $\begin{array}{l}37 \\
74\end{array}$ & $\begin{array}{l}31 \\
62\end{array}$ & $\begin{array}{l}19 \\
38\end{array}$ & - & - \\
\hline Sondagem & $\begin{array}{l}27 \\
54\end{array}$ & $\begin{array}{l}23 \\
46\end{array}$ & $\begin{array}{c}7 \\
14\end{array}$ & $\begin{array}{l}43 \\
86\end{array}$ & $\begin{array}{l}19 \\
38\end{array}$ & $\begin{array}{l}31 \\
62\end{array}$ & $\begin{array}{c}6 \\
12\end{array}$ & $\begin{array}{l}44 \\
88\end{array}$ & $\begin{array}{l}13 \\
26\end{array}$ & $\begin{array}{l}37 \\
74\end{array}$ & $\begin{array}{l}12 \\
24\end{array}$ & $\begin{array}{l}38 \\
76\end{array}$ & $\begin{array}{l}14 \\
28\end{array}$ & $\begin{array}{l}36 \\
72\end{array}$ & $\begin{array}{l}12 \\
24\end{array}$ & $\begin{array}{l}38 \\
76\end{array}$ & $\begin{array}{l}19 \\
38\end{array}$ & $\begin{array}{l}31 \\
62\end{array}$ & - & - \\
\hline $\begin{array}{l}\text { Manuseio de } \\
\text { drenos }\end{array}$ & $\begin{array}{l}46 \\
92\end{array}$ & $\begin{array}{l}4 \\
8\end{array}$ & $\begin{array}{l}11 \\
22\end{array}$ & $\begin{array}{l}39 \\
78\end{array}$ & $\begin{array}{l}31 \\
62\end{array}$ & $\begin{array}{l}19 \\
38\end{array}$ & $\begin{array}{l}14 \\
28\end{array}$ & $\begin{array}{l}36 \\
72\end{array}$ & $\begin{array}{l}22 \\
44\end{array}$ & $\begin{array}{l}28 \\
56\end{array}$ & $\begin{array}{l}18 \\
36\end{array}$ & $\begin{array}{l}31 \\
62\end{array}$ & $\begin{array}{l}27 \\
54\end{array}$ & $\begin{array}{l}23 \\
46\end{array}$ & $\begin{array}{l}14 \\
28\end{array}$ & $\begin{array}{l}36 \\
72\end{array}$ & $\begin{array}{l}35 \\
70\end{array}$ & $\begin{array}{l}15 \\
30\end{array}$ & - & - \\
\hline Curativos & $\begin{array}{l}45 \\
90\end{array}$ & $\begin{array}{c}5 \\
10\end{array}$ & $\begin{array}{l}11 \\
22\end{array}$ & $\begin{array}{l}39 \\
78\end{array}$ & $\begin{array}{l}33 \\
66\end{array}$ & $\begin{array}{l}17 \\
34\end{array}$ & $\begin{array}{l}14 \\
28\end{array}$ & $\begin{array}{l}36 \\
72\end{array}$ & $\begin{array}{l}17 \\
34\end{array}$ & $\begin{array}{l}33 \\
66\end{array}$ & $\begin{array}{l}20 \\
40\end{array}$ & $\begin{array}{l}30 \\
60\end{array}$ & $\begin{array}{l}33 \\
66\end{array}$ & $\begin{array}{l}17 \\
34\end{array}$ & $\begin{array}{l}13 \\
26\end{array}$ & $\begin{array}{l}37 \\
74\end{array}$ & $\begin{array}{l}36 \\
72\end{array}$ & $\begin{array}{l}14 \\
28\end{array}$ & - & - \\
\hline $\begin{array}{c}\text { Mudança de } \\
\text { decúbito }\end{array}$ & $\begin{array}{l}46 \\
92\end{array}$ & $\begin{array}{l}4 \\
8\end{array}$ & $\begin{array}{c}8 \\
16\end{array}$ & $\begin{array}{l}42 \\
84\end{array}$ & $\begin{array}{l}33 \\
66\end{array}$ & $\begin{array}{l}17 \\
34\end{array}$ & $\begin{array}{l}14 \\
28\end{array}$ & $\begin{array}{l}36 \\
72\end{array}$ & $\begin{array}{l}14 \\
28\end{array}$ & $\begin{array}{l}36 \\
72\end{array}$ & $\begin{array}{l}19 \\
38\end{array}$ & $\begin{array}{l}31 \\
62\end{array}$ & $\begin{array}{l}31 \\
62\end{array}$ & $\begin{array}{l}19 \\
38\end{array}$ & $\begin{array}{l}08 \\
16\end{array}$ & $\begin{array}{l}42 \\
84\end{array}$ & $\begin{array}{l}35 \\
70\end{array}$ & $\begin{array}{l}15 \\
30\end{array}$ & - & - \\
\hline
\end{tabular}

Legenda: - Não se aplica. Fonte: Dados da pesquisa (2021). 
No Quadro 1, foram pesquisados o uso dos seguintes EPIs: luvas de procedimento, máscara cirúrgica, máscara N95, máscara PFF, face shield, avental de tecido, avental descartável e óculos de proteção. Além disso, foi perguntado quanto à medida de distanciamento de um metro como maneira de proteção própria, a fim de reduzir as chances de contaminação com o novo coronavírus.

O uso dos referidos EPI foi verificado quanto a cada procedimento de enfermagem realizado. Os participantes puderam citar qual (is) EPI utilizavam para os seguintes procedimentos: admissão do paciente, banho, punção venosa, auxílio na intubação oro traquial (IOT), aspiração, administração de medicamentos, manuseio de drenos, mudança de decúbito e curativo.

No procedimento "Admissão dos pacientes", $90 \%$ dos profissionais entrevistados citaram utilizar luvas de procedimento, ao passo que $80 \%$ referiram fazer uso de óculos de proteção e $64 \%$ consideram a máscara de proteção respiratória com alta filtragem (N95) mais indicada. Seguido de $40 \%$ no que concerne ao uso de máscara cirúrgica e $30 \%$ na Peça Facial Filtrante (PFF) e paralelo, 50\% citaram utilizar simultaneamente o uso da face shield. Foi citado o uso do avental descartável na admissão dos pacientes por $72 \%$ dos participantes e em relação ao distanciamento, $82 \%$ não realizam.

Na categoria "Banho", $86 \%$ dos entrevistados mencionou o uso das luvas de procedimento, $68 \%$ empregam a máscara N95 e 58\% utilizam o avental descartável. O uso de óculos de proteção foi citado em $78 \%$ dos entrevistados.

Para a categoria "Mudança de decúbito", 92\% dos entrevistados referiram utilizar luvas de procedimento, 70\% óculos de proteção, 66\% a máscara N95 e 62\% o avental descartável. No que concerne o procedimento "Punção venosa", $92 \%$ dos profissionais fazem uso de luvas de procedimento, $64 \%$ demonstram preferência pela máscara N95, 58\% utilizam o avental descartável e $70 \%$ o uso dos óculos de proteção.

Durante o procedimento de "Auxílio na intubação orotraqueal", $60 \%$ dos entrevistados relatam o uso das luvas de procedimento, 36\% utilizam máscara N95 e 30\% a face shield. Contudo, números expressivos como $70 \%$ dos profissionais citaram não utilizar nenhum tipo de avental.

No procedimento de "Aspiração", 54\% fazem uso das luvas de procedimentos. Por outro lado, menos de 50\%, relatam utilizar algum tipo de máscara durante a assistência e o mesmo quantitativo se dá na questão da utilização dos aventais.

Quanto a "Mudança de decúbito do paciente", $92 \%$ relatam o uso das luvas de procedimento, $66 \%$ preferem utilizar a máscara N95. Outrossim, 62\% tem como primeira opção o avental descartável e 70\% fazem uso dos óculos de proteção.

Em relação ao procedimento "Administração de medicamentos", $84 \%$ relatam o uso das luvas de procedimento, $56 \%$ utilizam a máscara N95 e 62\% utilizam os óculos de proteção. Quanto a realização de "Sondagem", foi verificado que 54\% usam luvas de procedimentos, entretanto, mais de 60\% não utilizam nenhum tipo de máscara e óculos de proteção.

Já no procedimento "Manuseio de drenos", 92\% relataram fazer uso das luvas de procedimento, $62 \%$ optam pelo uso da máscara N95, 54\% utilizam o avental descartável e 70\% usam os óculos de proteção. Nos dados concernentes ao procedimento de "Curativo", 90\% dos profissionais afirmam utilizar luvas de procedimento, $66 \%$ faz uso da máscara N95 e com mesma proporção para o uso de avental descartável e 72\% utilizam os óculos de proteção.

Em relação ao "Distanciamento", como medida de proteção pessoal à contaminação, é necessário assinalar que os procedimentos estudados impossibilitam qualquer distância entre profissional e paciente durante a realização dos cuidados. Sendo assim, pontuou-se apenas o processo de admissão de paciente, em que $82 \%$ dos profissionais alegaram manter o afastamento. 


\section{Discussão}

Dentre os profissionais de enfermagem participantes do estudo, $14 \%$ são enfermeiros e a maior prevalência, $86 \%$, de técnicos de enfermagem. Segundo Camelo et al. (2013), os serviços de saúde, especificamente as unidades de cuidados intensivos exigem, atualmente, um profissional enfermeiro com um perfil que responda às necessidades de saúde dos pacientes. Desse modo, o resultado demonstra que a cada plantão na unidade possuía ao menos um profissional enfermeiro.

A maior proporção de técnicos de enfermagem, também é resultado em conformidade a Resolução nº 543 de 2017 do Conselho Federal de Enfermagem estabelece os parâmetros mínimos para dimensionar o quantitativo de profissionais de enfermagem. De acordo com a Resolução, um dos parâmetros baseia-se nas características do paciente em relação ao grau de dependência em relação a equipe de enfermagem e considera-se para cuidados de alta dependência, $36 \%$ de enfermeiros e demais técnicos e/ou auxiliares de enfermagem e para cuidado intensivo, 52\% enfermeiros e demais técnicos de enfermagem. Entretanto, ressalta Borges et al. (2017), que existem dificuldades na execução fidedigna desta distribuição, sendo uma prática comum o exercício profissional com a equipe com menos integrantes que mínimo previsto.

Quanto ao gênero dos participantes, foi evidenciado predominância de 88\% de mulheres, este achado é corroborado com outros estudos como de Camelo et al. (2013), realizado em UTI, e Martins et al. (2006). Para estes últimos autores, o fato reproduz a característica histórica da enfermagem, profissão exercida quase que exclusivamente por mulheres desde os seus primórdios. Destacamos este achado, a essência do cuidado humano, onde a figura feminina está ligada ao cuidar do outro, da família, caracterizando assim a profissão de enfermagem, ainda nos dias atuais, como essencialmente feminina.

Quanto a escolaridade dos profissionais de enfermagem, a maioria, 58\%, possui apenas o ensino médio completo, fato que pode ser compreendido tendo em vista que a maioria são técnicos de enfermagem. Contudo, $16 \%$ dos profissionais possuem pós-graduação, o que se configura como ponto positivo no desenvolvimento profissional e institucional. Resultado encontrado em estudo de Camelo et al. (2013), e os autores apontam a preocupação dos enfermeiros em buscar outras experiências além daquelas proporcionadas pelo ensino formal, que lhes possibilitem melhor preparo e maior segurança na prestação da assistência aos pacientes. Complementando com Lima et al. (2015), a educação continuada estimula o desenvolvimento da produtividade e competências específicas, além de gerar reconhecimento e motivação para o serviço. Nesse sentido, ressaltamos que a educação continuada deve ser estimulada por parte da instituição empregadora, assim como incentivo a realização de capacitações e aperfeiçoamentos.

A experiência profissional e o tempo em serviço foram tópicos levantados, e $34 \%$ dos profissionais revelaram ter mais de 5 anos de experiência e vínculo empregatício. Resultado que corrobora com estudo de Camelo et al. (2013), e apontam Martins et a.l (2006), a formação na graduação revela as competências e habilidades do enfermeiro, assim como o tempo de formação em uma dada época reflete o conhecimento e aptidão valorizados em um determinado período. Em vista disso, Almeida e Peres (2012), inferem que os profissionais com mais tempo em exercício profissional possuem habilidade como tomada de decisão, liderança, comunicação, resolução de conflitos, bem como maior familiaridade com as normas institucionais.

Em relação as informações e conhecimentos científicos a respeito da Covid-19, estão sendo realizadas em todos os lugares do mundo, inclusive no Brasil, o que poderá respaldar os profissionais de saúde que atuam na linha de combate na pandemia (Oliveira et al., 2021). Nesse sentido, o uso do EPI também deve ser e é considerado, por ser uma das medidas mais relevantes e adotadas neste momento de pandemia.

Nesse ínterim, Brasil (2020b) cita os EPI como de suma importância para proteção dos trabalhadores da enfermagem, em associação às medidas de precaução padrão. O empregador encarrega-se de disponibilizar esses equipamentos e garantir a quantidade correta e qualidade esperada para o seu uso, assim como a capacitação de seus colaboradores para o manuseio 
adequado dos mesmos, fazendo com que não haja desperdício desses materiais. O uso deve se dar de modo eficiente e racional, levando em conta o risco biológico de cada assistência prestada aos pacientes, assim como o descarte correto dos mesmos. Salienta-se que todos os EPI devem estar regidos pelos órgãos regulamentadores.

Dentre os EPI, a utilização das luvas de procedimento foi citada com mais de 50\% de uso em todos os procedimentos. Destaca-se a citação com mais $90 \%$ de uso nos procedimentos de admissão de pacientes, punção venosa, manuseio de dreno, curativos e mudança de decúbito. Entretanto, é preocupante a não adesão total dos profissionais nos procedimentos que requer o uso, isso revela o quanto é essencial a capacitação e qualificação dos profissionais de Enfermagem.

A luva de procedimento foi referida por $90 \%$ dos participantes na admissão de pacientes. Entretanto, segundo a Agência Nacional de Vigilância Sanitária (ANVISA) (2021), as luvas não se fazem necessárias no momento da admissão do paciente. Contudo, o Centro Estadual de Vigilância em Saúde, emitiu a Nota Informativa 01/2020 NVES/DVS/CEVS/SES, revisada em abril de 2020 e discorre sobre o uso das luvas no cenário pandêmico, e refere que devem ser empregadas quando o profissional for realizar assistência direta para com o paciente com suspeita e confirmados pelo Covid-19, para manipulação de materiais infectados por contato com secreções de pacientes, manejo de materiais e utensílios de pacientes suspeitos ou confirmados e quando a assistência possibilitar o contato com secreções, como, sangue, saliva, gotículas e aerossóis.

Nesse sentido, acredita-se que a utilização das luvas de procedimento na admissão pelos participantes do estudo se deve ao entendimento de estar prestando uma assistência ao paciente em seu recebimento no setor. Durante a admissão na UTI ou na enfermaria Covid, muitas vezes a equipe não tem as informações da real situação clínica do usuário, o que pode necessitar de uma intervenção imediata da Enfermagem.

Contudo, no procedimento de punção venosa, os dados coletados apontam que $92 \%$ dos profissionais aderem a utilização das luvas. Esse número não é suficiente, tendo em vista os riscos de contaminação que o procedimento apresenta. Segundo Santos et al. (2013), a utilização das luvas na técnica de punção venosa se faz de extrema importância, pois ela age como uma barreira de precaução, evitando assim contaminação por microrganismos, sendo que associada a higienização das mãos se torna o alicerce para precaução contra contaminação, e garante a seguridade para o profissional e paciente.

Ao analisar os dados sobre o uso das luvas de procedimento na administração de medicamentos, nota-se que $84 \%$ fazem o uso durante a administração de medicamentos, todavia há um percentual (16\%) que não citou utilizar esse EPI. Segundo o parecer Técnico Coren-SP $N^{\circ}$ 01/2021 o profissional só deve utilizar as luvas na administração de medicamentos mediante a observação da via a ser utilizada, associado às condições clínicas do paciente no momento da realização do procedimento, e considerar se essa administração poderá de alguma forma expor o profissional a contato com sangue e fluidos corpóreos. Entretanto, acreditamos que diante da pandemia, o uso das luvas deveria ser uma resposta unânime entre os participantes, visto que há risco de contaminação do profissional com contato direto com paciente.

No procedimento de Intubação orotraqueal, de acordo com a Associação Brasileira de Medicina de Emergência (ABRAMEDE) e Associação de Medicina Intensiva Brasileira (AMIB) (2020), a luva indicada a ser utilizada em tal assistência é a luva estéril. A enfermagem auxilia no procedimento, e os dados obtidos mostram que $60 \%$ fazem o uso das luvas de procedimento, até mesmo por auxiliar na fixação do tubo. Todavia, $40 \%$ dos participantes não referiram a utilização e o risco de contaminação é evidente, uma vez que a transmissão do coronavírus é por gotículas, saliva.

Na realização da aspiração orotraqueal, 54\% dos profissionais afirmam fazer uso das luvas de procedimento. Este fato diverge da literatura, uma vez que, segundo Martins et al. (2014), o procedimento de aspiração orotraqueal deve ser feito com técnica asséptica para a retirada passiva de secreções por um cateter conectado a um sistema de vácuo, introduzido na via aérea. 
Procedimentos de aerossolização, tais como, intubação orotraqueal, aspiração, coleta de amostra de Swab, entre outros, aumentam a chances de contato com gotículas e aerossóis e elevam o risco de contágio pelo novo coronavírus. Neste contexto, é de suma importância a utilização das luvas adequadas a cada procedimento, além da utilização dos demais EPI necessários em cada atividade exercida. Além disso, autores reforçam que em procedimentos que geram aerossóis, além das luvas de procedimento, os profissionais de saúde devem utilizar óculos de proteção ou protetor facial, máscara N95/PPF2 ou equivalente, avental, gorro e higienizar as mãos (WHO, 2020b; Carvalho et al., 2021).

Nesse contexto, em relação ao uso de máscara, a maior citação de uso foi a N95. Os procedimentos de maior adesão ao uso foram: admissão do paciente, banho, punção venosa, mudança de decúbito, administração de medicamentos, manuseio de drenos e curativos. Em segunda posição de citação de uso de máscara foi a PFF.

De acordo com Brasil (2020b), as máscaras N95 ou PFF devem ser usadas em procedimentos geradores de aerossóis (micronebulização, manobras de ressuscitação cardiopulmonar, intubação ou aspiração traqueal, coletas de amostras nasotraqueais, e diferentes procedimentos odontológicos) em pacientes com suspeita clínica ou confirmadas de Covid-19. Neste sentido, notamos que há coerência entre a utilização da máscara e o procedimento realizado. Além disso, para esses procedimentos a Organização Panamericana de Saúde (OPAS) (2020) orienta a utilização de aventais cirúrgicos, luvas e óculos ou face shield.

Em relação aos tipos de avental, tecido, descartável e impermeável, o de maior citação foi o descartável. Os procedimentos com citações acima de 50\% do uso desse tipo de avental foram: admissão, banho, punção venosa, manuseio de dreno e curativo. O uso de avental impermeável deve ser utilizado, se o profissional estiver atuando em uma unidade com quartos Covid, assim como para sair de uma acomodação para outra (SS; DEVISA, 2020).

Torna-se relevante evidenciar o fato de que mais de 50\% dos participantes da pesquisa referiram fazer uso do EPI, como luvas de procedimento, máscara N95 e avental descartável nos procedimentos de admissão, banho, punção venosa, mudança de decúbito manuseio de dreno e curativos. Isso revela o conhecimento que possuem quanto à realização dos procedimentos que geram aerossóis e quanto ao contato com paciente suspeito ou confirmado de Covid-19. Achado importante, pois nesse hospital de estudo logo que instaurada a pandemia, fora realizada conforme referem Couto et al. (2020), ação educativa acerca da paramentação e desparamentação de EPI no contexto da Covid-19 para a equipe de enfermagem, a fim de incentivar a realização de ações de maneira segura aos trabalhadores. No resultado foi unanime a resposta quanto a contribuição da ação para o crescimento do conhecimento e solicitaram novas educações sobre o tema. Nesse sentido, revela que o conhecimento apreendido está sendo colocado em prática.

Todavia, notou-se em alguns procedimentos que a utilização do EPI precisa ser mais aderida entre os profissionais, uma vez que menos de 50\% relataram o uso de máscara e avental em procedimentos, como auxílio na intubação oro traqueal e aspiração. Fato preocupante diante do panorama de crescimento de casos da Covid-19 e forte potencial para contaminação. Autores como Santana et al. (2020), referem que alguns fatores como, o anseio pelo uso de EPI apropriado, de forma correta e ao fato de que estes ao cobrirem o corpo todo protegem mais, mas que em geral são do tipo desconfortáveis, de material sintético, impermeável e, comumente mais complexos para pôr e retirar, são situações que podem colaborar para a baixa ou não adesão aos protocolos de combate a Covid-19.

Neste sentido, corroboramos com Garcia et al. (2021), ao referir que é imprescindível a capacitação dos profissionais de saúde quanto ao uso do EPI com técnicas de paramentação e retirada destes, a fim de evitar possível contaminação. E, especialmente durante a desparamentação, deve-se ter bastante precaução em razão de ser mais propícia ao contágio pelo vírus (Santana et al., 2020). Acrescentam-se Couto et al. (2020), que incentivam a realização de ações educativas, e que estas sejam inovadoras para estimular os participantes. 
Quanto aos demais cuidados junto ao paciente, como a mudança de decúbito e realização de curativos, a OPAS (2020) orienta a utilização de máscara cirúrgica, avental cirúrgico, luvas e óculos ou face shield. Positivamente, o exposto pode ser observado na pesquisa, na qual os profissionais ao realizar curativo utilizam, luvas, máscara (neste momento a de maior referência foi a N95), avental descartável e óculos de proteção, assim protegendo-se de contaminação.

No tocante ao distanciamento durante a assistência, verifica-se a prevalência de mais de $80 \%$ dos profissionais que não mantém a distância mínima de um metro durante a assistência. Tal fato, impera devido a necessidade de permanecer próximo ao paciente para realizar os cuidados de enfermagem. Esta medida deve ser adotada para a vida social, em outra atividade que não seja no momento de assistência ao paciente. Contudo, deve ser utilizada a máscara cirúrgica para evitar a contaminação da boca e nariz do profissional por gotículas respiratórias, quando o mesmo atuar a uma distância inferior a um metro do paciente suspeito ou confirmado de infecção pelo novo coronavírus (Soares et al., 2020).

Evidentemente, o uso de EPI é apenas uma medida entre diversas que compõem a hierarquia de medidas de controle da Covid-19 (Santana et al., 2020). Considera-se que, além do uso adequado de EPI, fatores como a organização do trabalho, o estabelecimento de fluxo de pessoas no ambiente contaminado e limpo, o uso de técnicas e protocolos são essenciais para a proteção dos profissionais de saúde e dos pacientes no ambiente hospitalar e podem, quando utilizados em conjunto, prevenir adoecimento e mortes desses profissionais (Garcia et al., 2021).

Complementa-se aos ambientes de trabalho, os controles de engenharia e arquitetura no combate à Covid-19 e, dentre as medidas, estão barreiras físicas como coberturas protetoras em macas, assentos e outros componentes da estrutura, instalação de obstáculos, como placas de vidros, acrílicas ou janelas para atendimento administrativo. Ademais, os aspectos de barreiras, ambientes, equipamentos, instalações, materiais, resíduos e fluidos de serviços de saúde são fatores relevantes a serem ponderados para o processo de trabalho diante da pandemia (Santana et al., 2020).

Como limitação do presente estudo é o fato de refletir apenas a realidade local do hospital estudado. Assim sendo, deve-se reproduzir técnicas de coletas de dados in loco a fim de conhecer a adequação às orientações produzidas pelos principais órgãos de saúde pública nacional e internacional em outros locais de assistência à saúde.

\section{Conclusão}

Diante do cenário mundial de infecção pelo coronavírus, este estudo possibilitou caracterizar os profissionais atuantes como protagonistas no combate à pandemia por Covid-19 e conhecer a realidade local quanto ao uso do EPI pelos profissionais de enfermagem. Os achados mostram de modo geral que os profissionais utilizam os EPI nos diversos procedimentos e os de maior citação de uso foram: luvas de procedimento, máscara N95 e avental descartável. Entretanto, em alguns procedimentos, a utilização do EPI precisa ser aderida entre os profissionais.

$\mathrm{Na}$ oportunidade, cabe ao enfermeiro verificar e prover os insumos necessários para a assistência segura ao profissional, garantindo a disponibilidade dos equipamentos bem como verificar o correto manuseio, paramentação, desparamentação e descarte destes. Além disso, vale ressaltar que os EPI são somente uma das medidas efetivas dentro de um conjunto de medidas que inclui controles administrativos, ambientais e de engenharia.

Assim sendo, torna-se imprescindível a realização de ações de educação permanente em saúde, a fim de garantir a adesão dos profissionais às medidas de controle da doença.

\section{Referências}

Agência Nacional de Vigilância Sanitária. (2021). Nota Técnica GVIMS/GGTES/ANVISA nº 04/2020. Orientações para serviços de saúde: medidas de prevenção e controle que devem ser adotadas durante a assistência aos casos suspeitos ou confirmados de infecção pelo novo coronavírus (SARS-CoV-2) atualizada em 25/02/2021. Brasília, Distrito Federal. https://www.gov.br/anvisa/pt-br/centraisdeconteudo/publicacoes/servicosdesaude/notas-tecnicas/notatecnica-gvims_ggtes_anvisa-04_2020-25-02-para-o-site.pdf/view 
Almeida, M. D. L., \& Peres, A. M. (2012). Conhecimentos, habilidades e atitudes sobre a gestão dos formados de Enfermagem de uma universidade pública brasileira. Investigación y Educación en Enfermería, 30(1), 66-76. http://www.scielo.org.co/pdf/iee/v30n1/v30n1a08.pdf

Antunes, C.M.T.B.; Luciano, C.C.; Bahia, J.C.; Araújo, R.M.; Bastos, R.M.A.F.P. (2021). Relato de experiência dos atendimentos de Enfermagem em triagem para o diagnóstico da COVID-19 em profissionais da saúde. Nursing, 24(277), 5785-5792. http://revistas.mpmcomunicacao.com.br/index.php/revistanursing/article/view/976/1120

Associação Brasileira de Medicina de Emergência, Associação de Medicina Intensiva Brasileira, Sociedade Brasileira de Cardiologia, \& Conselho LatinoAmericano de Emergências Cardiovasculares e Ressuscitação. (2020). Recomendações para Intubação Orotraqueal em pacientes portadores de COVID-19. Sociedade Brasileira de Cardiologia. https://www.portal.cardiol.br/post/recomenda\%C3\%A7\%C3\%B5es-para- intuba\%C3\%A7\%C3\%A3o-orotraqueal-empacientes-portadores-de-covid-19

Brasil. Ministério do Trabalho e Previdência. (2020a) NR 06 - Equipamento de Proteção Individual. Brasília: Ministério do Trabalho e Previdência. https://www.gov.br/trabalho-e-previdencia/pt-br/composicao/orgaos-especificos/secretaria-de-trabalho/inspecao/seguranca-e-saude-no-trabalho/ctppnrs/norma-regulamentadora-no-6-nr-6

Brasil. Ministério da Saúde. (2020b). Recomendações de proteção aos trabalhadores dos serviços de saúde no atendimento de COVID-19 e outras síndromes gripais. Brasília, DF. https://www.saude.go.gov.br/files/banner_coronavirus/GuiaMS-Recomendacoesdeprotecaotrabalhadores-COVID-19.pdf.

Borges, F., Bohrer, C. D., Bugs, T. V., Nicola, A. L., Tonini, N. S., de Oliveira, J. L. C. (2017). Dimensionamento de pessoal de Enfermagem na UTI-Adulto de hospital universitário público. Cogitare Enfermagem, 22(2). http://dx.doi.org/10.5380/ce.v22i2.50306.

Carvalho, A. A. G., Aidar, A. L., Santos, B. C. D., Kuramoto, D. A. B., Pereda, M. R., Correia, R. M., Amorim, J. E. (2021). Recomendações de uso de equipamentos de proteção individual (EPIs) em procedimentos cirúrgicos durante a pandemia de SARS-Cov. Jornal Vascular Brasileiro, 20. https://doi.org/10.1590/1677-5449.200044

Camelo, S. H. H., Silva, V. L. S., Laus, A. M., Chaves, L. D. P. (2013). Perfil profissional de enfermeiros atuantes em Unidades de Terapia intensiva de um hospital de ensino. Ciencia y Enfermeria XIX, (3): 51-62. https://scielo.conicyt.cl/pdf/cienf/v19n3/art_06.pdf

Centro Estadual de Vigilância em Saúde. Divisão de Vigilância Sanitária. Núcleo de Vigilância de Estabelecimentos de Saúde. (2020). Nota informativa 01/2020 NVES/DVS/CEVS/SES. Orientação aos profissionais de saúde e à população para o uso de máscaras de proteção e luvas de procedimentos como forma de prevenção e controle de infecções pelo novo coronavírus (COVID-19). Centro Estadual de Vigilância Sanitária, Porto Alegre, RS https://saude.rs.gov.br/upload/arquivos/202004/07101549-nota-informativa-uso-mascaras-e-luvas-revisada-em-06-04-2020.pdf.

Conselho Federal de Enfermagem. (2017). Resolução COFEN n 543, de 2017. Atualiza e estabelece parâmetros para o dimensionamento do quadro de profissionais de Enfermagem nos serviços/locais em que são realizadas atividades de Enfermagem. Diário Oficial, Brasília, DF. http://www.cofen.gov.br/resolucao-cofen-5432017_51440.html

Conselho Regional de Enfermagem de São Paulo. Câmara Técnica. (2021). Parecer Técnico COREN-SP no 01/2021. Uso de Equipamento de Proteção Individual (EPI) na sala de vacinação. Conselho Regional de Enfermagem de São Paulo. https://portal.coren-sp.gov.br/wpcontent/uploads/2021/01/PARECER- T\%C3\%89CNICO-N\%C2\%BA-01-2021.pdf

Couto, M. C., Migueis, G. S., Teodoro, C. J., Santos, K. O., Alves, K. M. S. Oliveira, W. J., Oliveira, W. S. (2020, janeiro). Ação educativa: uso dos equipamentos de proteção individual durante a pandemia de Covid-19. Anais da VII Mostra Científica de Enfermagem e do III Seminário da Pós-Graduação em Saúde. Rondonópolis, MT. https//www.even3.com.br/anais/mostraenf/300032-ACAO-EDUCATICA--USO-DOS-EQUIPAMENTOS-DE-PROTECAOINDIVIDUAL-DURANTE-A-PANDEMIA-DE-COVID-19.

Garcia, G. P. A., Fracarolli, I. F. L., Santos, H. E. C. D., Souza, V. R. D. S., Cenzi, C. M., Marziale, M. H. P. (2021). Utilização de equipamentos de proteção individual para atendimento de pacientes com covid-19: revisão de escopo. Revista Gaúcha de Enfermagem, 42. https://doi.org/10.1590/19831447.2021.20200150

Knechtel, M. D. R. (2014). Metodologia da pesquisa em educação: uma abordagem teórico-prática dialogada. Curitiba: Inter Saberes. https://dtcom.com.br/wayco/temas/section_2/pesquisa_qualitativa_e_quantitativa/sections/pdf/THEME4285.pdf

Lima, A. S., Nicolato, F. V., Dutra, H. S., Bahia, M. T. R., Farah, B. F. (2015). A educação permanente na gestão da atenção primária de saúde no Sistema Único de Saúde. Revista de Enfermagem UFPE, 9(4). https://periodicos.ufpe.br/revistas/revistaEnfermagem/article/download/10569/11507.

Martins, C., Kobayashi, R. M., Ayoub, A. C., Leite, M. M. J. (2006). Perfil do enfermeiro e necessidades de desenvolvimento de competência profissional.Texto Contexto Enferm, Jul-Set; 15(3): 472-8. https://pdfs.semanticscholar.org/5735/12b5611ca4222f695b741cd70ea279a0bc99.pdf

Martins, R., Nunes, P. M., Xavier, P. A., Wittkopf, P. G., Schivinski, C. I. S. (2014). Aspiração traqueal: a técnica e suas indicações. ACM Arquivos Catarinenses de Medicina, 90-96. http://www.acm.org.br/revista/pdf/artigos/1280.pdf

Oliveira, W. S., Migueis, G. S., Silva, M. S., Oliveira, W. J. (2021). Conhecimento sobre Covid-19 dos profissionais de Enfermagem atuantes no enfrentamento da doença. Research, Society and Development, 10(11).

Organização Pan-Americana da Saúde. (2020). Transmissão do SARS-CoV-2: implicações para as precauções de prevenção de infecção. https://iris.paho.org/bitstream/handle/10665.2/52472/OPASWBRACOVID-1920089_por.pdf?sequence=1\&isAllowed=y

Secretaria de Saúde (SS).; Departamento de Vigilância em Saúde (DEVISA). (2020). Equipamentos de proteção individual no contexto do Covid-19, de acordo com o tipo de ambiente, profissionais alvo e tipo de atividade. Medidas de controle para prevenção da infecção pelo novo coronavírus - Covid-19. Prefeitura de Campinas, SS, DEVISA.https://covid-19.campinas.sp.gov.br/sites/covid-19.campinas.sp.gov.br/files/recomendacoestecnicas/Conte\%C3\%BAdo\%2020\%20-\%20EPI\%20no\%20contexto\%20da\%20COVID-

19\%20de $\% 20$ acordo\%20com\%20o\%20tipo\%20de\%20ambiente\%20profissionais\%20alvo\%20e\%20tipo\%20de\%20atividade.\%20Edi\%C3\%A7\%C3\%A3o\%2 02\%20-\%2013\%20de\%20maio\%20de\%202020.pdf 
Research, Society and Development, v. 11, n. 2, e4011225447, 2022

(CC BY 4.0) | ISSN 2525-3409 | DOI: http://dx.doi.org/10.33448/rsd-v11i2.25447

Santana, N., Costa, G. A., Costa, S. D. S. P., Pereira, L. V., Silva, J. V. D., Sales, I. P. P. M. (2020). Segurança dos profissionais de saúde no enfrentamento do novo coronavírus no Brasil. Escola Anna Nery, 24. https://www.scielo.br/j/ean/a/qzdy4jvzyRck6FfxMBGL4mh/?lang=pt

Santos, R., Roseira, C. E., Batista Dias Passos, I. P., de Figueiredo, R. M. (2013). O uso de luvas pela equipe de Enfermagem: da proteção ao risco de transmissão. Revista de Enfermagem UFPE, 7(11).

Soares, S. S. S., de Oliveira Souza, N. V. D., Silva, K. G., César, M. P., Souto, J. D. S. S., Pereira, J. C. R. A. (2020). Pandemia de Covid-19 e o uso racional de equipamentos de proteção individual. Revista Enfermagem UERJ, 28.

Teixeira, C. F. D. S., Soares, C. M., Souza, E. A., Lisboa, E. S., Pinto, I. C. D. M., Andrade, L. R. D., Espiridião, M. A. (2020). A saúde dos profissionais de saúde no enfrentamento da pandemia de Covid-19. Ciência \& Saúde Coletiva, 25, 3465-3474. https://www.epublicacoes.uerj.br/index.php/Enfermagemuerj/article/view/50360

Tristão, F. S. İ. A., Tavares, D. H. (2020). Equipamentos de proteção individual para atendimento de casos suspeitos ou confirmados do novo Coronavírus. Journal of Nursing and Health, 20104042-20104042. https://pesquisa.bvsalud.org/controlecancer/resource/pt/biblio-1145285?src=similardocs

World Health Organization. (2020a). Rational use of personal protective equipment for coronavirus disease (COVID-19): interim guidance. https://apps.who.int/iris/handle/10665/331215

World Health Organization (2020b). Infection prevention and control during health care when novel coronavirus (nCoV) infection is suspected: interim guidance. https://www.who.int/publications/i/item/10665-3314 